\title{
Randomized, Sham Controlled Trial of Transcranial Direct Current Stimulation for Painful Diabetic Polyneuropathy
}

\author{
Yon Joon Kim, $\mathrm{MD}^{1}$, Jeonghun Ku, $\mathrm{PhD}^{2}$, Hyun Jung Kim, MD ${ }^{1}$, Dal Jae Im, MD¹, \\ Hye Sun Lee, $\mathrm{MS}^{3}$, Kyung Ah Han, $\mathrm{MD}^{4}$, Youn Joo Kang, MD
}

${ }^{1}$ Department of Rehabilitation Medicine, Eulji Hospital, Eulji University School of Medicine, Seoul; ${ }^{2}$ Department of Biomedical Engineering, Keimyung University, Daegu; ${ }^{3}$ Department of Biostatistics, Yonsei University College of Medicine, Seoul; ${ }^{4}$ Department of Medicine, Eulji Hospital, Eulji University School of Medicine, Seoul, Korea

Objective To investigate the analgesic effect of transcranial direct current stimulation (tDCS) over the primary motor (M1), dorsolateral prefrontal cortex (DLPFC), and sham tDCS in patients with painful diabetic polyneuropathy (PDPN).

Methods Patients with PDPN ( $n=60$ ) were divided randomly into the three groups ( $\mathrm{n}=20$ per group). Each group received anodal tDCS with the anode centered over the left M1, DLPFC, or sham stimulation for 20 minutes at intensity of $2 \mathrm{~mA}$ for 5 consecutive days. A blinded physician rated the patients' pain using a visual analog scale (VAS), Clinical Global Impression (CGI) score, anxiety score, sleep quality, Beck Depression Inventory (BDI), and the pain threshold (PT) to pressure.

Results After the tDCS sessions, the M1 group showed a significantly greater reduction in VAS for pain and PT versus the sham and DLPFC groups $(\mathrm{p}<0.001)$. The reduction in VAS for pain was sustained after 2 and 4 weeks of follow-up in the M1 group compared with the sham group $(\mathrm{p}<0.001, \mathrm{p}=0.007)$. Significant differences were observed among the three groups over time in VAS for pain $(\mathrm{p}<0.001)$, CGI score $(\mathrm{p}=0.01)$, and PT $(\mathrm{p}<0.001)$. No significant difference was observed among the groups in sleep quality, anxiety score, or BDI score immediately after tDCS.

Conclusion Five daily sessions of tDCS over the M1 can produce immediate pain relief, and relief 2- and 4-week in duration in patients with PDPN. Our findings provide the first evidence of a beneficial effect of tDCS on PDPN.

Keywords Transcranial direct current stimulation, Chronic pain, Diabetic neuropathy

Received May 31, 2013; Accepted August 19, 2013

Corresponding author: Youn Joo Kang

Department of Rehabilitation Medicine, Eulji Hospital, 68 Hangeulbiseong-ro, Nowon-gu, Seoul 139-711, Korea

Tel: +82-2-970-8315, Fax: +82-2-979-8268, E-mail: md52516@hanmail.net

(ㄷ) This is an open-access article distributed under the terms of the Creative Commons Attribution Non-Commercial License (http://creativecommons. org/licenses/by-nc/3.0) which permits unrestricted noncommercial use, distribution, and reproduction in any medium, provided the original work is properly cited.

Copyright $\odot 2013$ by Korean Academy of Rehabilitation Medicine

\section{INTRODUCTION}

Diabetes mellitus (DM) is a chronic disease causing injury from the peripheral nerves to the brain. About $30 \%$ of diabetic patients experience neuropathic pain [1]. Painful diabetic polyneuropathy (PDPN) is characterized by persistent pain that substantially affects the quality of life of diabetic patients. It can lead to moderate-to-severe 
unremitting lower limb pain in over $70 \%$ of sufferers [2]. Not surprisingly, those affected experience a reduction in their daily activities and loss of employment, which can result in profound depression and poor quality of life [3]. The management of PDPN can be challenging for the clinician and the patient because the pain is often unresponsive, or only partially responsive to existing pharmacological approaches [4].

Previous research on PDPN has focused on peripheral nervous system dysfunction. Injury to peripheral nerves causes functional and biochemical changes at the site of injury [5]. However, recent studies have suggested that central neuropathic mechanisms can also contribute to the pain experienced with diabetes [6]. Furthermore, recent studies revealed that the central nervous system (CNS) is involved during even in the early stages of PDPN [7]. During the progression of PDPN, higher-order neurons within the spinal cord and brain are affected [8]. Advances in neuroimaging methods have resulted in a better understanding of how PDPN affects the CNS. Magnetic resonance spectroscopy has demonstrated thalamic dysfunction in patients with DM [9]. In experimental models, neurons in the ventral posterolateral thalamus can become hyperexcitable, firing at abnormally high frequencies and generating aberrant spontaneous activity [10].

Non-invasive brain stimulation (NBS) may modulate neuropathic pain that is refractory medical treatment. Repetitive transcranial magnetic stimulation (rTMS), cranial electrotherapy stimulation, and transcranial direct current stimulation (tDCS) are effective in reducing neuropathic pain [11]. In tDCS, the cerebral cortex is noninvasively and painlessly stimulated using a weak direct current in non-invasive and painless manner. Furthermore, tDCS may have some advantages in that it may lead to longer-lasting modulatory effects on cortical function, is easy to administer and perform, less expensive, and provides a reliable sham-stimulation condition to assess the specificity of the effects compared with other methods $[11,12]$.

Several trials have demonstrated that tDCS applied over the primary motor cortex (M1) may relieve chronic pain in spinal cord injury, trigeminal neuralgia, and multiple sclerosis [13-15]. To our knowledge, no previous study has used tDCS in PDPN and no consensus about the appropriate electrode position has been reached. Thus, we sought to assess whether anodal tDCS stimulation on M1 or the dorsolateral prefrontal cortex (DLPFC) is associated with a clinical reduction of pain and other benefits, such as improvements in physical function, anxiety, and depression, as compared with sham stimulation in patients with PDPN. The M1 and DLPFC were chosen as targets because previous studies revealed that stimulation of M1 induces a significant analgesic effect, and DLPFC stimulation is associated with a significant analgesic or anti-depressive effect $[16,17]$.

\section{MATERIALS AND METHODS}

\section{Subjects}

Seventy two patients with type $2 \mathrm{DM}$ presenting with chronic, drug-resistant neuropathic pain were enrolled. All patients were selected from inpatient services at the Diabetes Mellitus Center of the Department of Endocrinology at our hospital. They were regarded as suitable to participate if they fulfilled the following criteria: 1) DM diagnosis by blood sugar levels [18], 2) PDPN diagnosis by electrodiagnostic tests or neuropathy total symptom score $($ NTSS) $>6$ [19], 3 ) score $\geq 4$ or higher ( $0=$ 'no pain' and $10=$ 'worst possible pain') on a visual analog scale (VAS) for pain perception at treatment baseline, 4) stable chronic pain for at least 3 preceding months, and 5) persistent pain after taking medications, such as non-steroidal anti-inflammatory drugs, tramadol, antidepressants, antiepileptic drugs, or opioids.

We excluded patients with PDPN who had 1) any uncontrolled clinical disease (as evaluated by each patient's clinician), such as thyroid, cardiovascular, pulmonary, hematological, pulmonary, or renal disease or psychiatric disorder; 2) history of substance abuse or neuropsychiatric comorbidity; 3) implanted devices for pain control, such as vagal or deep brain stimulators; 4) pain attributable to other causes, such as spinal stenosis, peripheral blood vessel disease, lower extremity edema-related pain, and neuropathic pain due to a brain lesion or peripheral nerve injury; 5) contraindication for tDCS, such as an intracranial or orbital metallic implant or pacemaker; 6) Parkinson disease; 7) alcoholism; and/or 8) severe cognitive deficit on the Mini-Mental Status Examination (MMSE) score <24).

Patients who were receiving medication for pain were not excluded. Pharmacological therapies that showed 
no or limited positive effects or side effects at high dosages were observed in all patients. We selected patients receiving stable doses of analgesics for at least 2 months prior to the beginning of this study. No changes in the medication regimens for pain were permitted throughout the trial.

All patients who consented to participate in this study were informed about tDCS and the experimental protocol, which was approved by the Institutional Review Board of our hospital.

The sample size for a mixed model (repeated-measures analysis of variance [ANOVA]) was calculated according to the primary end points. We considered three groups and four time repetitions to detect differences by VAS. A sample size of 51 participants was sufficient to detect an effect value of 0.25 (Cohen's medium effect size) and correlation among repeated measures of 0.3 at a significance level of 0.05 (two-sided) with $90 \%$ power, using the G*Power software (ver. 3.0.10; http://www.psycho.uniduesseldorf.de/abteilungen/aap/gpower3). We thus determined a sample size of 72 participants, considering a $30 \%$ drop-out rate.

\section{Experimental design}

The randomized, sham-controlled, single-center trial was designed to evaluate the efficacy and safety of tDCS sessions in PDPN. All patients were divided into three groups: M1, sham, and DLPFC (Fig. 1). Randomization was performed using the order of entry into the study and a computer-generated randomization chart with random blocks of six patients each. This study consisted of a 5-day period of tDCS treatment; the number of stimulation sessions was established on the basis of previous studies that showed the efficacy of tDCS in the treatment of other causes of chronic pain $[13,20]$. Patients and the assessing physicians were blinded to group allocation, whereas the treating physician, who set the tDCS according to the protocol, was aware of the stimulation condition. To minimize communication among patients, the treating physician was instructed not to talk to patients or the assessing physicians regarding the experiment protocol.

This study had three phases: 1 ) baseline evaluation consisting of the administration of a VAS for pain, Clinical Global Impression (CGI), BDI, and pain threshold (PT) using a pressure algometer; 2) a period of daily treatment sessions with tDCS for 5 consecutive days; and 3) followup evaluations conducted after 2 and 4 weeks. The assessing physician conducted baseline evaluations of all patients, including the measurement of glycated hemoglobin (HbAlc) concentration, calculation of the NTSS score [19], determination of the durations of DM and PDPN, MMSE score, and collection of the medication

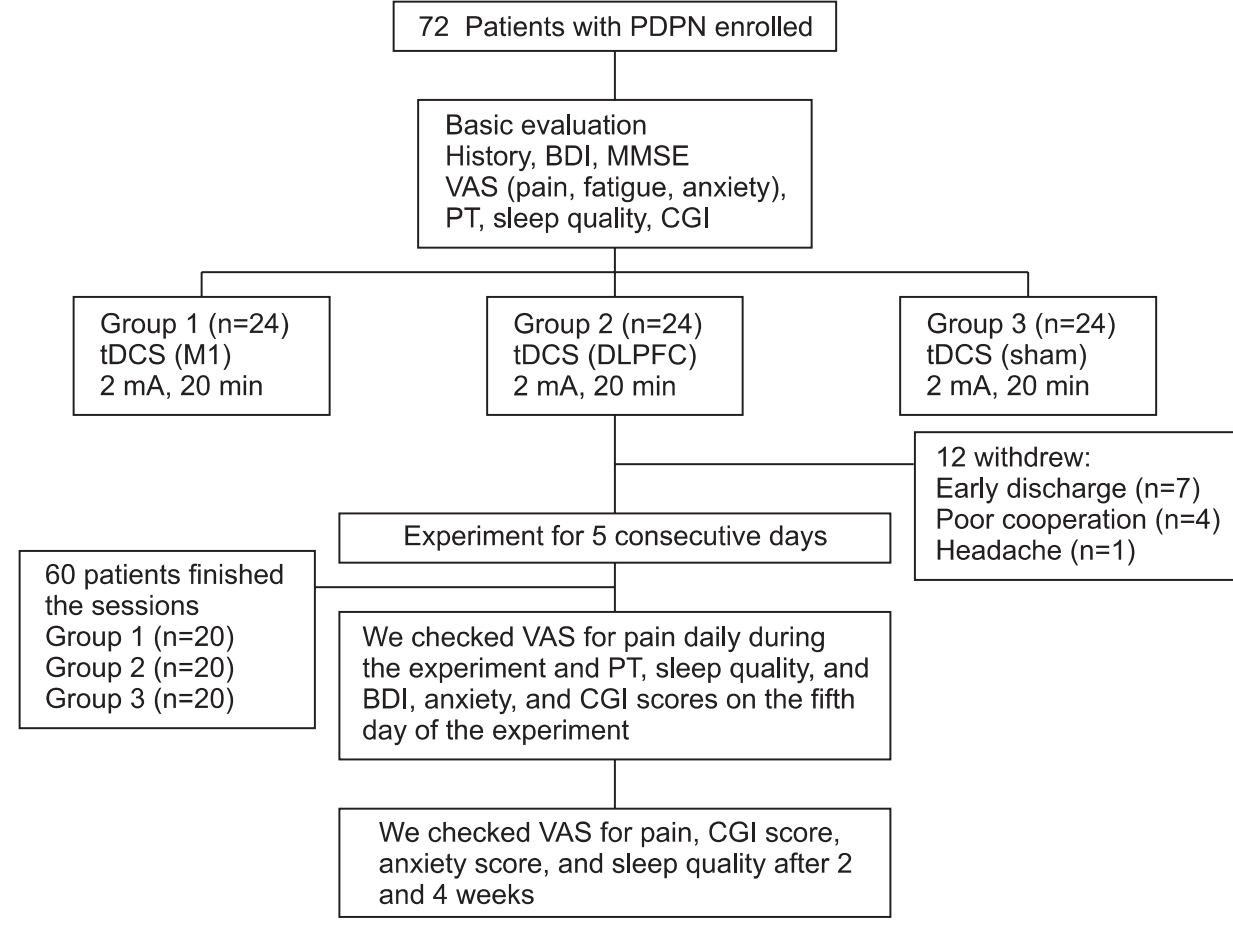

Fig. 1. Patient flow diagram. Twelve patients withdrew from the study: seven had early discharge during stimulation sessions and five withdrew after the third session of stimulation because of poor cooperation $(n=4)$ or headache $(n=1)$. PDPN, painful diabetic polyneuropathy; BDI, Beck Depression Index; MMSE, Mini-Mental State Examination; VAS, visual analog scale; PT, pain threshold; CGI, Clinical Global Impression; tDCS, transcranial direct current stimulation; M1, primary motor cortex; DLPFC, dorsolateral prefrontal cortex. 
history for pain (type and mean dose). The assessing physicians also administered the VAS for pain daily after the tDCS session, determined CGI, anxiety, and BDI scores, assessed sleep quality in terms of subject reported total sleep time and number of awakenings during sleep [21], and measured the PT after 5 days of tDCS sessions. VAS for pain, CGI, anxiety score, and sleep quality were also assessed after 2 and 4 weeks by telephone. The CGI reflects the severity of illness and is rated on a seven-point scale ranging from 1 to 7 , where 1 indicates normal (not ill) and 7 indicates the most severe illness. The severity of illness item requires the physician to rate the severity of a patient's illness at the time of assessment relative to the physician's past experience with patients who had the same diagnosis [22]. We measured the domains of depression using BDI and VAS for anxiety because both can be important confounders in pain improvement. The BDI is a 21-item test presented in multiple-choice format, which measures the presence and the degree of depression in adults [23]. The VAS for anxiety is a self evaluation scale ranging from 0 to 10 , where 0 indicates no anxiety and 10 indicates the worst possible anxiety.

\section{tDCS}

A Phoresor II PM850 anodal tDCS was used (IOMED, Salt Lake City, UT, USA). For M1 anodal stimulation, the anode (saline-soaked electrodes, $5 \times 5 \mathrm{~cm}$ ) was placed over C3 (EEG 10/20 system) and the cathode over the contralateral supraorbital area (Fig. 2). For anodal stimulation in the DLPFC group, the anode was placed over F3 (EEG $10 / 20$ system) and the cathode over the contralateral supraorbital area. This method of DLPFC localization has been used and has been confirmed to be relatively accurate by neuro-navigation [24,25]. A constant current with an intensity of $2 \mathrm{~mA}$ was used for a single 20 -minute session. This protocol is safe and effective in patients with neuropathic pain [13]. For sham stimulation, the same electrode positions were used as in anodal M1 stimulation, but the stimulator was on for only 30 seconds. The patients felt the initial itching sensation but received no current for the rest of the stimulation period. This method of sham stimulation is reliable [12,14].

\section{Evaluation of PT}

The blinded assessing physician evaluated PT before and after five sessions of tDCS. PT recordings involved applying an increasing amount of blunt pressure using the $1 \mathrm{~cm}^{2}$ hard rubber end of a Commander algometer (JTECH Medical Industries, Salt Lake City, UT, USA). Several discrete pressures were applied successively to the more painful (right or left) sole region at an approximate rate of $2 \mathrm{lb} / \mathrm{s}$ until the subject reported perceiving pain, at which point the device was removed and the PT value was recorded [26]. This procedure was repeated three times and then averaged for analysis. If patients complained of pain equally on both soles, PT was checked on the right or left side randomly three times and the values were averaged.

\section{Adverse effects}

Patients were queried after each session of tDCS and in the follow-up whether they had experienced adverse effects and how these effects were related to the tDCS treatment. Known adverse effects during and/or after application of tDCS are a tingling sensation, moderate fatigue, a light itching sensation under the stimulation electrodes, headache, nausea, and insomnia [27].

\section{Statistical analyses}

For statistical analyses, a linear mixed model for a repeated-measures covariance pattern model with unstructured covariance within subjects was used. Two

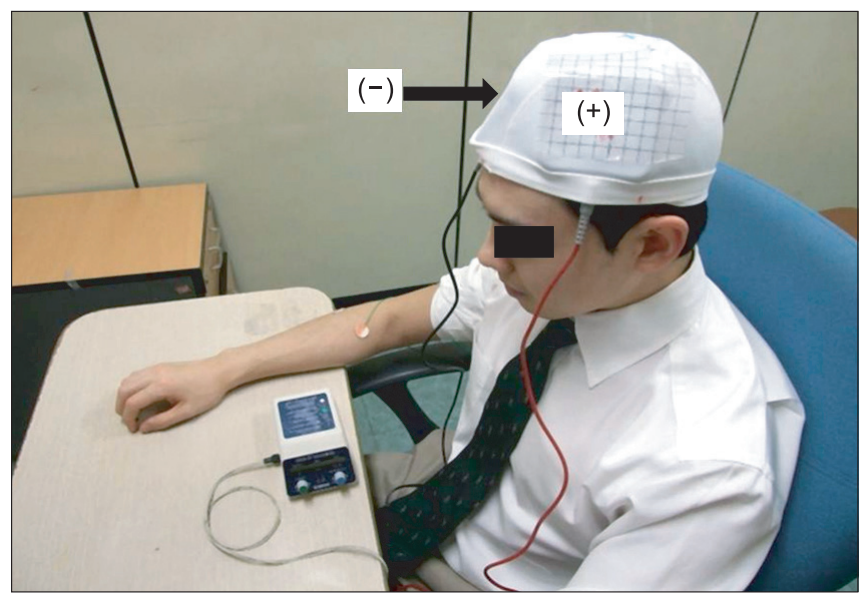

Fig. 2. Anodal tDCS. The anode was positioned over the M1 or DLPFC and the cathode over the contralateral supraorbital region ( $2 \mathrm{~mA}$ for 20 minutes on 5 consecutive days). tDCS, transcranial direct current stimulation; M1, primary motor cortex; DLPFC, dorsolateral prefrontal cortex. 
fixed effects were included: one between-subjects group effect (M1, sham, and DLPFC) and one within-subject time effect (four times: baseline, 5 days, 2 weeks, and 4 weeks). Possible difference variables (VAS for pain, CGI, anxiety score, sleep quality, BDI score, and PT) in the groups across time were analyzed according to time $\times$ group interactions. Likewise, dates of maximal pain reduction (VAS for pain) were analyzed among subjects to determine time effects at eight time points (baseline; 1, 2, 3,4 , and 5 days; and 2 and 4 weeks) using a mixed model. When a significant difference among the three groups was noted across time, a post-hoc Bonferroni multiplecomparisons test was also used. We divided the three groups into two categories according to reduction in VAS for pain on the fifth day ( $>30 \%$ and $<30 \%$ ) and performed chi-square tests to identify correlations among the three groups. We also used one-way ANOVA for baseline comparisons among the three groups $(\mathrm{p}<0.05)$ and Student t-test for comparisons of analgesic effect and other baseline characteristics (age, sex, pain duration, baseline PT and baseline BDI, anxiety, and CGI scores). All data were analyzed using the SAS ver. 9.2 software (SAS Institute, Cary, NC, USA). Unless stated otherwise, all results are presented as mean \pm standard deviation and statistical significance refers to a two-tailed p-value $<0.05$.

\section{RESULTS}

Twelve (15\%) subjects dropped out during the experiment (four each in the M1, DLPFC, and sham groups).

Table 1. Clinical and demographic characteristics of the 60 subjects

\begin{tabular}{|c|c|c|c|c|}
\hline Characteristic & $\begin{array}{c}\text { M1 tDCS } \\
(\mathrm{n}=\mathbf{2 0})\end{array}$ & $\begin{array}{c}\text { Sham tDCS } \\
(n=20)\end{array}$ & $\begin{array}{c}\text { DLPFC tDCS } \\
(n=20)\end{array}$ & p-value \\
\hline Gender, male & $9(45)$ & $8(40)$ & $8(40)$ & \\
\hline Age (yr) & $59.60 \pm 13.15$ & $61.60 \pm 10.27$ & $63.50 \pm 8.75$ & 0.848 \\
\hline Duration of DM (yr) & $14.00 \pm 7.42$ & $15.75 \pm 7.97$ & $13.45 \pm 6.32$ & 0.286 \\
\hline NTSS & $9.78 \pm 2.70$ & $9.29 \pm 1.75$ & $9.30 \pm 1.76$ & 0.671 \\
\hline \multicolumn{5}{|l|}{ Pain location } \\
\hline Hand & 4 & 4 & 3 & \\
\hline Foot & 20 & 20 & 20 & \\
\hline Both & 4 & 4 & 3 & \\
\hline \multicolumn{5}{|l|}{ Duration of pain (yr) } \\
\hline$>5$ & 10 & 10 & 11 & \\
\hline $2-5$ & 10 & 10 & 9 & \\
\hline$<2$ & 0 & 0 & 0 & \\
\hline \multicolumn{5}{|l|}{ Baseline } \\
\hline VAS for pain & $5.75 \pm 0.71$ & $5.55 \pm 0.78$ & $5.70 \pm 0.73$ & 0.692 \\
\hline BDI score & $10.60 \pm 2.95$ & $11.10 \pm 3.55$ & $8.75 \pm 1.94$ & 0.774 \\
\hline PT (Ib) & $3.19 \pm 0.70$ & $3.29 \pm 0.62$ & $3.44 \pm 0.52$ & 0.993 \\
\hline CGI & $4.45 \pm 0.99$ & $4.70 \pm 1.13$ & $4.35 \pm 1.09$ & 0.507 \\
\hline HbAlc (\%) & $9.81 \pm 1.95$ & $9.84 \pm 2.15$ & $9.80 \pm 1.68$ & 0.998 \\
\hline MMSE & $27.00 \pm 2.69$ & $27.05 \pm 2.12$ & $27.30 \pm 2.32$ & 0.204 \\
\hline \multicolumn{5}{|l|}{ Fasting BST (mg/dL) } \\
\hline 1st day ${ }^{\mathrm{a})}$ & $137.75 \pm 39.95$ & $131.10 \pm 36.32$ & $134.05 \pm 39.93$ & 0.858 \\
\hline 5th day ${ }^{b)}$ & $135.40 \pm 39.30$ & $129.00 \pm 31.69$ & $130.10 \pm 19.91$ & 0.667 \\
\hline
\end{tabular}

Values are presented as mean \pm standard deviation or number (\%).

tDCS, transcranial direct current stimulation; M1, primary motor cortex; DLPFC, dorsolateral prefrontal cortex; DM, diabetes mellitus; NTSS, Neuropathy Total Symptom Score; VAS, visual analog scale; BDI, Beck Depression Inventory; PT, pain threshold; CGI, Clinical Global Impression; MMSE, Mini-Mental State Examination; BST, blood sugar test. ${ }^{a)}$ BST at baseline, ${ }^{\text {b) }} \mathrm{BST}$ at the end of tDCS sessions. 
The reasons were early discharge in seven patients, poor cooperation in four, and headache in one patient. Sixty patients underwent five consecutive tDCS treatment sessions with no significant adverse effects. The mean ages of the three groups were $59.6 \pm 13.15$ years in the M1 group, $61.6 \pm 10.27$ years in the sham group, and $63.5 \pm 8.75$ years in the DLPFC group (Table 1).

No baseline difference among the three groups was observed in terms of age, sex, duration of DM, duration of pain, HbAlc concentration, severity of NTSS, VAS for pain, PT, medication characteristics, or BDI, CGI, or MMSE score (Tables 1, 2). The most common sites of pain were the lower extremities $(100 \%)$, upper extremities (18.3\%), and both (18.3\%). No changes were evident in the three groups concerning changes in fasting glucose levels on day 1 and 5 of tDCS application (Table 1). The patients were given routine medical treatment according to the guidelines of our pain clinic using pregabalin (150$300 \mathrm{mg})$, gabapentin (600-1,200 mg), and thioctic acid (225-600 mg). Other medications used included NSAIDs (51.66\%) and tricyclic antidepressants (40\%). No patient was taking morphine (Table 2). No significant differences in medication was found among the three groups.

\section{Improvement in VAS pain score after tDCS treatment}

Results from the mixed model showed a significant interaction in time versus group for pain reduction $\left(\mathrm{F}_{(6,57)}=8.96, \mathrm{p}<0.001\right)$. Post-hoc comparisons showed that the $\mathrm{M} 1$ group had greater pain reduction than the sham $(\mathrm{t}=6.46, \mathrm{p}<0.001)$ and DLPFC $(\mathrm{t}=5.39, \mathrm{p}<0.001)$ groups after tDCS sessions. Furthermore, the M1 group showed greater pain reduction than the sham group after 2 weeks $(\mathrm{t}=4.07, \mathrm{p}<0.001)$ and 4 weeks $(\mathrm{t}=3.17, \mathrm{p}=0.007)$ of the follow-up period (Fig. 3). Likewise, the M1 group showed greater pain reduction than the DLPFC group $(t=3.64$, $\mathrm{p}=0.002)$ after 2 weeks.

Baseline VAS scores for pain in the $\mathrm{M} 1$ group decreased from $5.750 \pm 0.716$ to $3.800 \pm 0.523$, and $33.91 \%(20 \%-50 \%)$ of maximal pain reduction occurred after the fifth tDCS session. In the sham group, the baseline VAS score for pain was $5.550 \pm 0.759$ and pain decreased after the tDCS sessions to $4.800 \pm 0.615 ; 13.51 \%(0 \%-33.3 \%)$ of pain reduction was reported after tDCS sessions. In the DLPFC group, the baseline VAS score for pain was $5.700 \pm 0.732$ and it decreased after tDCS sessions to $4.450 \pm 0.759$; $21.93 \%(0 \%-33.3 \%)$ of pain reduction compared with baseline was reported. At the time of the fifth tDCS ses-

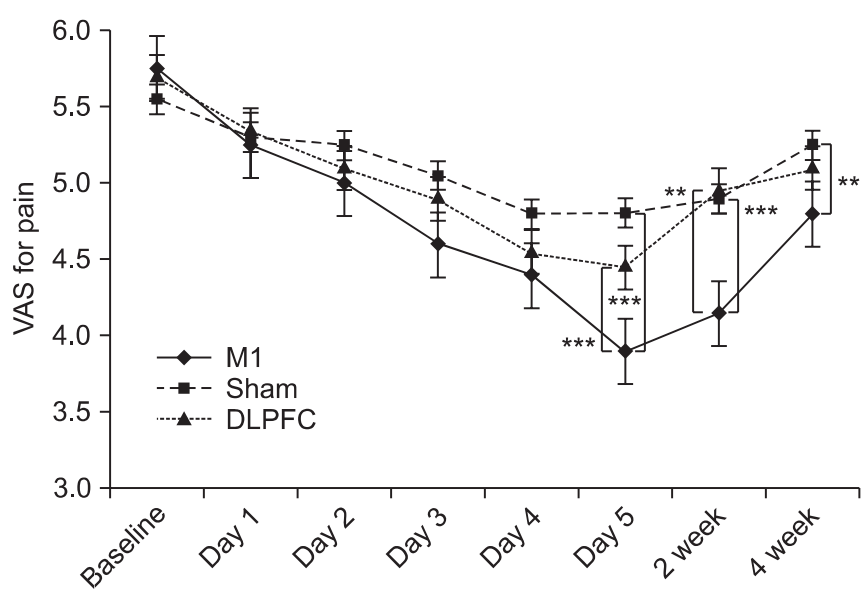

Fig. 3. After 5 consecutive days of tDCS sessions over M1, pain reduction was significantly greater compared with the sham and DLPFC groups $\left({ }^{* * *} \mathrm{p}<0.001\right)$ and compared with the sham group, its analgesic effect was sustained after 2 weeks $\left({ }^{* * *} p<0.001\right)$ and 4 weeks $\left({ }^{* *} p<0.01\right)$ of follow-up. Likewise, the M1 group showed greater pain reduction than the DLPFC group $\left({ }^{* *} \mathrm{p}<0.01\right)$ after 2 weeks. tDCS, transcranial direct current stimulation; M1, primary motor cortex; DLPFC, left dorsolateral prefrontal cortex; VAS, visual analog scale.

Table 2. Medications used in the study

\begin{tabular}{|c|c|c|c|c|c|c|}
\hline \multirow{2}{*}{ Drug } & \multicolumn{2}{|c|}{ M1 tDCS } & \multicolumn{2}{|c|}{ Sham tDCS } & \multicolumn{2}{|c|}{ DLPFC tDCS } \\
\hline & No. (\%) & Dose & No. (\%) & Mean dose & No. (\%) & Dose \\
\hline Gabapentin & $0(0)$ & - & $2(10)$ & $1,050.00 \pm 212.10$ & $2(10)$ & $600.00 \pm 0$ \\
\hline Thioctic acid & $4(20)$ & $550.00 \pm 100$ & $4(20)$ & $506.25 \pm 187.50$ & $4(20)$ & $600.00 \pm 0$ \\
\hline Pregabalin & $6(30)$ & $162.50 \pm 30.62$ & $5(25)$ & $210.00 \pm 62.75$ & $5(25)$ & $175.00 \pm 38.73$ \\
\hline Amitriptyline & $4(20)$ & $10.00 \pm 0$ & $5(25)$ & $10.00 \pm 0$ & $5(25)$ & $10.00 \pm 0$ \\
\hline Nortriptyline & $4(20)$ & $10.00 \pm 0$ & $3(15)$ & $10.00 \pm 0$ & $4(20)$ & $10.00 \pm 0$ \\
\hline
\end{tabular}

Values are presented as mean \pm standard deviation.

tDCS, transcranial direct current stimulation; M1, primary motor cortex; DLPFC, dorsolateral prefrontal cortex. 
sion, $13 / 20(65 \%)$ subjects in the M1 group, but only one patient (5\%) in the sham group and seven (35\%) in the DLPFC group, reported $\geq 30 \%$ pain reduction. This difference across the three groups was statistically significant $(\mathrm{p}<0.001)$.

We analyzed whether the effect of pain reduction was related to age, pain duration, body mass index, baseline PT, and/or baseline BDI, anxiety, and CGI scores. We divided all patients into two categories: $>30 \%$ and $<30 \%$ reduction in VAS score for pain $(n=21$ and $n=39$, respectively). Pain reduction did not differ significantly according to baseline characteristics ( $p>0.05$ ).

\section{CGI scale}

The baseline CGI score in the M1 group was $4.45 \pm 0.99$; after five sessions of tDCS, the CGI score decreased to $3.05 \pm 0.99$. The CGI score decreased compared with baseline by about $31.46 \%(0 \%-80 \%)$ in the M1 group. In the sham group, the baseline CGI score was $4.7 \pm 1.13$ and this score decreased to $4.35 \pm 0.74$ after the tDCS sessions. A $7.45 \%(0 \%-20 \%)$ change in the CGI score from baseline was observed. In the DLPFC group, the baseline CGI score was $4.35 \pm 1.09$ and this score decreased to $3.45 \pm 1.05$ after the tDCS sessions, representing a $20.68 \%(0 \%-40 \%)$ reduction from baseline. A significant time versus group

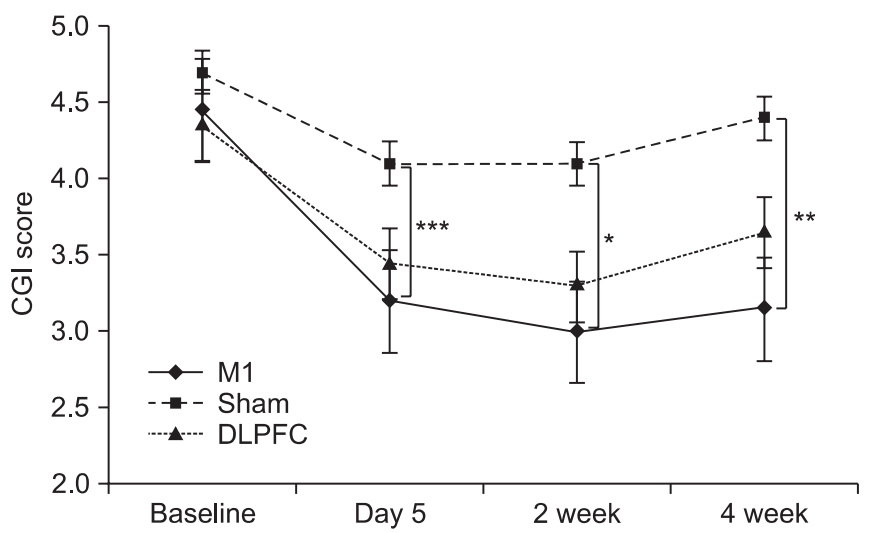

Fig. 4. After 5 consecutive days of tDCS sessions, the M1 group exhibited a significantly greater reduction in CGI score $\left({ }^{* * *} \mathrm{p}<0.001\right)$, which was decreased significantly after 2 and 4 weeks of follow-up compared with the sham group $\left({ }^{*} \mathrm{p}=0.02,{ }^{* *} \mathrm{p}=0.008\right)$. CGI is rated on a 7 -point scale: 1 , normal, not ill at all; 2 , borderline mentally ill; 3 , mildly ill; 4 , moderately ill; 5 , markedly ill; 6 , severely ill; 7 , most severely ill. tDCS, transcranial direct current stimulation; M1, primary motor cortex; DLPFC, left dorsolateral prefrontal cortex; CGI, Clinical Global Impression. interaction was observed for CGI change $\left(F_{(6,57)}=2.96\right.$, $\mathrm{p}=0.01$ ). Post-hoc comparison showed that the $\mathrm{Ml}$ group had a significantly greater CGI score reduction after tDCS sessions ( $\mathrm{t}=4.01, \mathrm{p}<0.001)$, and at 2 weeks $(\mathrm{t}=2.87, \mathrm{p}=0.02)$ and 4 weeks $(\mathrm{t}=3.16, \mathrm{p}=0.008)$ in the follow-up period, versus the sham group. No significant difference in the change in CGI score was noted between the Ml and DLPFC or the sham and DLPFC groups (Fig. 4).

\section{PT to pressure}

The baseline PT in the M1 group was $3.19 \pm 0.70 \mathrm{lb} / \mathrm{s}$ and PT increased to $3.41 \pm 0.73 \mathrm{lb} / \mathrm{s}$ after five sessions of tDCS. PT increased about $6.44 \%(0 \%-11.53 \%)$ relative to baseline. In the sham group, baseline PT was $3.27 \pm 0.59 \mathrm{lb} / \mathrm{s}$ and PT increased to $3.36 \pm 0.56 \mathrm{lb} / \mathrm{s}$ after tDCS sessions, a $2.67 \%(-2.32 \%$ to $7.40 \%)$ PT change relative to baseline. In the DLPFC group, baseline PT was $3.44 \pm 0.52 \mathrm{lb} / \mathrm{s}$ and it increased to $3.55 \pm 0.52 \mathrm{lb} / \mathrm{s}$ after the tDCS sessions, a $2.25 \%(-2.77 \%$ to $8.57 \%)$ change relative to baseline. Results from the mixed model showed a significant time versus group interaction for change in $\mathrm{PT}\left(\mathrm{F}_{(2,57)}=15.44\right.$, $\mathrm{p}<0.001)$. Post-hoc comparisons showed a significantly greater increase in PT in the M1 group than in the DLPFC group $(\mathrm{t}=5.41, \mathrm{p}<0.001)$ or the sham group $(\mathrm{t}=-3.81$, $\mathrm{p}<0.001$ ) after the tDCS sessions (Fig. 5).

\section{Anxiety, sleep characteristics, and BDI score}

Although significant anxiety score changes were observed among the three groups over time $\left(\mathrm{F}_{(6,57)}=2.84\right.$,

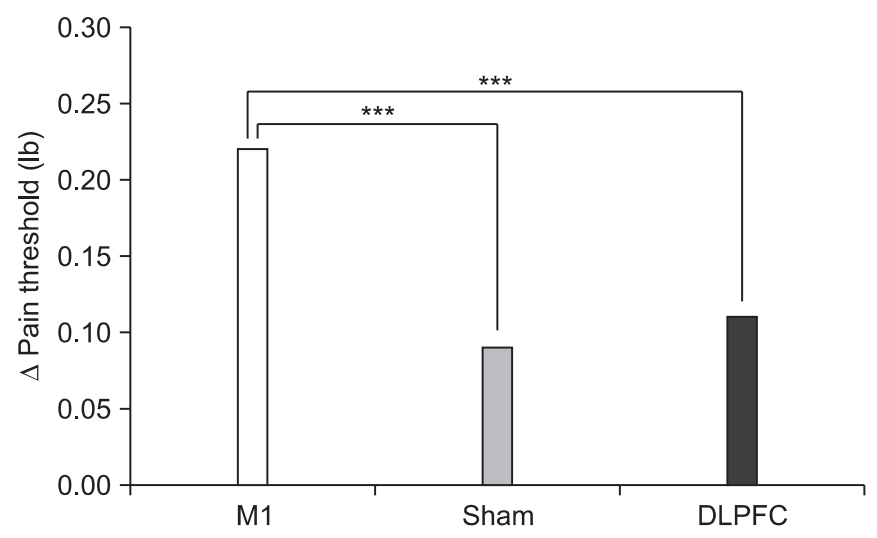

Fig. 5. After 5 consecutive days of tDCS sessions, the M1 group exhibited a significantly greater increase in pain threshold compared with the sham and DLPFC groups $\left({ }^{* * *} \mathrm{p}<0.001\right)$. tDCS, transcranial direct current stimulation; M1, primary motor cortex; DLPFC, left dorsolateral prefrontal cortex. 
interaction $\mathrm{p}=0.02)$, post-hoc comparisons revealed no difference in the immediate change in anxiety score after five tDCS sessions between the M1 and sham ( $\mathrm{t}=1.15$, $\mathrm{p}=0.77)$ or $\mathrm{M} 1$ and DLPFC $(\mathrm{t}=1.37, \mathrm{p}=0.52)$ groups (Table $3)$. The M1 group showed significantly greater reductions in anxiety scores than did the sham group after 2 and 4 weeks during the follow-up period $(\mathrm{p}=0.005, \mathrm{t}=3.28$; $\mathrm{p}=0.03, \mathrm{t}=2.62$ ).

Changes in sleep quality were assessing by total sleep time and number of awakenings. No significant change in total sleep time $\left(\mathrm{F}_{(6,57)}=0.86\right.$, interaction $\left.\mathrm{p}=0.53\right)$ or number of awakenings $\left(\mathrm{F}_{(6,57)}=0.85\right.$, interaction $\left.\mathrm{p}=0.54\right)$ was observed among the three groups over time (Table 3 ).

We assessed BDI scores in all subjects before tDCS and after five sessions of tDCS. In the Ml group, baseline BDI was $10.60 \pm 2.94$ and it was $9.55 \pm 2.35$ after tDCS. In the sham group, baseline BDI was $11.10 \pm 3.55$ and it was 10.10 \pm 3.12 after tDCS. In the DLPFC group, baseline BDI was $8.75 \pm 1.94$ and it was $7.75 \pm 1.65$ after tDCS. After 5 days of tDCS sessions, changes in BDI score did not differ significantly among the three groups $\left(\mathrm{F}_{(2,57)}=0.03\right.$, interaction $\mathrm{p}=0.97$ ) (Table 3).

\section{Adverse effects}

All participants tolerated tDCS well without experiencing any significant adverse effects. Six incidents of adverse events occurred in the three groups. The adverse events were headache (three patients: two in the M1 group and one in the DLPFC group) and itching under the electrodes (three patients: one in each group).

\section{DISCUSSION}

The results presented here show that in patients with
PDPN, anodal tDCS at the M1 significantly improved pain and PT to pressure versus DLPFC and sham stimulation. This effect was specific to the stimulation site and lasted for up to 4 weeks after treatment stopped. In addition, pain reduction was not associated with baseline characteristics including age, pain duration, BMI, baseline BDI score, baseline anxiety score, baseline CGI score, or baseline PT ( $\mathrm{p}>0.05)$.

Potential analgesic effects of tDCS at M1 were evident in patients with PDPN. The findings are consistent with studies that showed that anodal tDCS effectively improves pain in patients with other types of chronic pain syndrome, such as those from spinal cord injury, multiple sclerosis, or trigeminal neuralgia [13-15,20]. The effects of tDCS on the pain processing network system are poorly understood in patients with chronic neuropathic pain, and the present study provides no direct evidence to clarify these effects. In patients with chronic central pain, increased local excitability at M1 may be associated with pain control. Motor cortex stimulation has been a treatment option for patients with chronic neuropathic pain for more than 15 years [28]. Furthermore, an animal study indicated that excitatory electrical stimulation over M1 may directly or indirectly modulate the activity of the thalamus and convey an inhibitory influence from the M1 [29]. Functional magnetic resonance imaging has revealed that high-frequency rTMS over M1 induces significant deactivation in remote nociceptive brain structures and increases the sensory perception threshold in healthy volunteers [30]. Modulation of M1 with highfrequency rTMS or anodal tDCS has been proposed to result in the inhibition of hyperactivity in areas that underlie chronic pain including the medial thalamus, anterior cingulate cortex, and upper brain stem [31]. Further

Table 3. Comparison of anxiety, depression, and sleep quality after five sessions of tDCS

\begin{tabular}{|c|c|c|c|c|c|c|}
\hline & \multicolumn{2}{|c|}{ M1 vs. Sham } & \multicolumn{2}{|c|}{ M1 vs. DLPFC } & \multicolumn{2}{|c|}{ Sham vs. DLPFC } \\
\hline & $\begin{array}{l}\text { Estimated } \\
\text { mean (SE) }\end{array}$ & p-value ${ }^{\text {a) }}$ & $\begin{array}{l}\text { Estimated } \\
\text { mean (SE) }\end{array}$ & p-value ${ }^{a)}$ & $\begin{array}{l}\text { Estimated } \\
\text { mean (SE) }\end{array}$ & p-value ${ }^{a}$ \\
\hline Anxiety score & $0.25(0.22)$ & 0.77 & $0.30(0.23)$ & 0.52 & $0.05(0.22)$ & $>0.99$ \\
\hline BDI score & $0.05(0.32)$ & $>0.99$ & $-0.03(0.32)$ & $>0.99$ & $-0.08(0.32)$ & $>0.99$ \\
\hline Total sleep time & $-0.23(0.18)$ & 0.64 & $0.05(0.18)$ & 2.35 & $0.28(0.18)$ & 0.39 \\
\hline Awakenings $^{\text {b) }}$ & $0.10(0.17)$ & $>0.99$ & $<0.01$ & $>0.99$ & $-0.10(0.17)$ & $>0.99$ \\
\hline
\end{tabular}

tDCS, transcranial direct current stimulation; M1, primary motor cortex; DLPFC, dorsolateral prefrontal cortex; BDI, Beck Depression Inventory; SE, standard error.

${ }^{a)} \mathrm{p}$-value was corrected by Bonferroni method, ${ }^{\text {b) }}$ number of awakenings during sleep. 
studies are needed to provide deeper insight about the nociceptive mechanisms of tDCS in chronic pain.

Interestingly, our findings showed that tDCS at M1 provided superior pain reduction in PDPN compared with DLPFC. We found no significant analgesic effect or change in PT after tDCS over DLPFC. DLPFC has been suggested to play important roles in anxiety, depression, and unpleasantness related to pain [32]. Additionally, DLPFC may be activated during painful states and may, in turn, ultimately modulate structures involved in the emotional perception of pain including the anterior cingulate cortex, insula, and amygdala [33]. In patients with major depression, 10-Hz TMS of the left DLPFC reduces pain [34]. M1 stimulation may produce an analgesic effect by affecting sensory aspects of pain, whereas DLPFC stimulation may affect pain-associated affective-emotional networks. We did not confirm the analgesic effects of tDCS only in depressive patients in the DLPFC group because most patients had low BDI scores (11.33 \pm 3.67$)$.

We evaluated the off-line after-effects of repeated tDCS sessions over different brain sites. The analgesic effects lasted for 2 and 4 weeks after five tDCS sessions at M1, but not in the DLPFC or sham group. These findings are consistent with those of a previous study of 17 patients with spinal cord injuries where the analgesic effect was sustained for 2 weeks after five sessions of daily tDCS sessions at M1 [13]. Likewise, after five sessions of tDCS at M1, the analgesic effect was sustained after 3 weeks in 32 patients with fibromyalgia [20]. Anodal tDCS is associated with increased cortical excitability that lasts beyond the stimulation period [31]. tDCS over M1 is known induces widespread and long-lasting analgesic effects in neural networks $[17,32]$.

In this study, a $33.91 \%(20 \%-50 \%)$ mean degree of pain reduction was achieved after five tDCS sessions. Other rTMS and tDCS studies reported analgesic effects in patients with chronic pain ranging from $20 \%$ to $58 \%$ $[13,15,33]$. Additionally, cathodal tDCS of the visual cortex reduced the duration of attacks and the intensity of pain in patients with migraine [34]. We can assume that the analgesic effect of tDCS for chronic pain is related to the type of pain syndrome, type of equipment, method of stimulation (electrode position, polarity, duration, intensity), and site of pain. To achieve the maximal analgesic effect, further studies are needed to provide an optimal stimulation paradigm of tDCS for various pain syndromes.

In this study, the PT was increased by $6.44 \%\left(0 \%^{-}\right.$ $11.53 \%)$ in the M1 group and $2.25 \%(-2.77 \%$ to $8.57 \%)$ in the DLPFC group. Boggio et al. [17] showed that the PT increased by $8.3 \%$ and $10 \%$ after tDCS over M1 and DLPFC, respectively, in healthy volunteers. Moreover, they proposed that the synchronized stimulation of M1 and DLPFC may confer a greater increase in the PT than either stimulation alone. In another study, cathodal tDCS of the primary sensory cortex significantly reduced the sensitivity to A $\delta$-fiber-mediated cold sensation [35]. We can assume that the tDCS effect on pain perception is related to the condition of the subject, stimulation parameters of the equipment, and type of stimulation (pressure, laser, electrical, cold, and warm). Further studies are needed to clarify these effects.

In this study, only a low incidence of minor adverse effects, such as mild headache or itching (8.33\%), occurred in comparison with previous tDCS studies. One patient dropped out during the experiment due to minor adverse effects (headache). In other studies that used tDCS for patients with fibromyalgia and those with spinal cord injury, 11/32 (34.37\%) and 9/17 (52.94\%) patients, respectively, reported adverse events, although they were benign and minor $[13,20]$.

This study has some limitations. First, we did not exclude patients who were receiving medication for pain. We minimized the interaction effect between medication and tDCS across the three groups as follows. The mean doses and numbers of pain medications did not differ across the three groups. In addition, we selected patients receiving stable doses of analgesics for at least 2 months before the beginning of this study and allowed no change in medication regimens for pain throughout the trial. Second, the tDCS electrode has a relatively large surface area $\left(25 \mathrm{~cm}^{2}\right)$, and the analgesic effect or effect on PT of tDCS can be mediated by action on the somatosensory cortex. Further studies are needed to clarify whether more focal and somatotopically guided tDCS stimulation might enhance the analgesic effect. Another limitation is that we measured only the somatosensory PT and did not assess other types of PT. Laser stimuli activate A $\delta$ and $\mathrm{C}$ pain fibers, whereas pressure stimuli activate primarily A $\beta$ fibers [36].

The findings of the current study support the need for future investigations of novel neuromodulatory ap- 
proaches for the treatment of PDPN. To maximize and provide sustained analgesic effects, further studies that aim to optimize parameters of NBS for PDPN, such as equipment type and the intensity and polarity of stimulation, are needed. Furthermore, additional studies are needed to provide deeper insight about the nociceptive mechanisms of tDCS in chronic pain.

In conclusion, the findings provide evidence that five daily sessions of anodal tDCS over the M1, but not sham tDCS or anodal tDCS over the DLPFC, effectively reduce pain and increase the PT in patients with PDPN. The approach can produce pain relief of 2-4 weeks and improved physical function in patients with PDPN. This study provides preliminary evidence for the beneficial effects of tDCS in PDPN.

\section{CONFLICT OF INTEREST}

No potential conflict of interest relevant to this article was reported.

\section{ACKNOWLEDGMENTS}

This paper was supported by Eulji University in 2012 (EJRG-12-014-042B81).

\section{REFERENCES}

1. Boulton AJ, Malik RA, Arezzo JC, Sosenko JM. Diabetic somatic neuropathies. Diabetes Care 2004;27:1458-86.

2. Davies M, Brophy S, Williams R, Taylor A. The prevalence, severity, and impact of painful diabetic peripheral neuropathy in type 2 diabetes. Diabetes Care 2006;29:1518-22.

3. Jensen MP, Chodroff MJ, Dworkin RH. The impact of neuropathic pain on health-related quality of life: review and implications. Neurology 2007;68:1178-82.

4. Tesfaye S, Chaturvedi N, Eaton SE, Ward JD, Manes C, Ionescu-Tirgoviste $\mathrm{C}$, et al. Vascular risk factors and diabetic neuropathy. N Engl J Med 2005;352:341-50.

5. Baron R. Mechanisms of disease: neuropathic pain: a clinical perspective. Nat Clin Pract Neurol 2006;2:95106.

6. Fischer TZ, Waxman SG. Neuropathic pain in diabetes: evidence for a central mechanism. Nat Rev Neurol 2010;6:462-6.
7. Selvarajah D, Wilkinson ID, Emery CJ, Harris ND, Shaw PJ, Witte DR, et al. Early involvement of the spinal cord in diabetic peripheral neuropathy. Diabetes Care 2006;29:2664-9.

8. Eaton SE, Harris ND, Rajbhandari SM, Greenwood P, Wilkinson ID, Ward JD, et al. Spinal-cord involvement in diabetic peripheral neuropathy. Lancet 2001;358:35-6.

9. Selvarajah D, Wilkinson ID, Emery CJ, Shaw PJ, Griffiths PD, Gandhi R, et al. Thalamic neuronal dysfunction and chronic sensorimotor distal symmetrical polyneuropathy in patients with type 1 diabetes mellitus. Diabetologia 2008;51:2088-92.

10. Fischer TZ, Tan AM, Waxman SG. Thalamic neuron hyperexcitability and enlarged receptive fields in the STZ model of diabetic pain. Brain Res 2009;1268:15461.

11. Fregni F, Freedman S, Pascual-Leone A. Recent advances in the treatment of chronic pain with noninvasive brain stimulation techniques. Lancet Neurol 2007;6:188-91.

12. Gandiga PC, Hummel FC, Cohen LG. Transcranial DC stimulation (tDCS): a tool for double-blind shamcontrolled clinical studies in brain stimulation. Clin Neurophysiol 2006;117:845-50.

13. Fregni F, Boggio PS, Lima MC, Ferreira MJ, Wagner T, Rigonatti SP, et al. A sham-controlled, phase II trial of transcranial direct current stimulation for the treatment of central pain in traumatic spinal cord injury. Pain 2006;122:197-209.

14. Mori F, Codeca C, Kusayanagi H, Monteleone F, Buttari F, Fiore S, et al. Effects of anodal transcranial direct current stimulation on chronic neuropathic pain in patients with multiple sclerosis. J Pain 2010;11:43642.

15. Khedr EM, Kotb H, Kamel NF, Ahmed MA, Sadek R, Rothwell JC. Longlasting antalgic effects of daily sessions of repetitive transcranial magnetic stimulation in central and peripheral neuropathic pain. J Neurol Neurosurg Psychiatry 2005;76:833-8.

16. Fregni F, Boggio PS, Nitsche MA, Marcolin MA, Rigonatti SP, Pascual-Leone A. Treatment of major depression with transcranial direct current stimulation. Bipolar Disord 2006;8:203-4.

17. Boggio PS, Zaghi S, Lopes M, Fregni F. Modulatory effects of anodal transcranial direct current stimulation 
on perception and pain thresholds in healthy volunteers. Eur J Neurol 2008;15:1124-30.

18. American Diabetes Association. Diagnosis and classification of diabetes mellitus. Diabetes Care 2010;33 Suppl 1:S62-9.

19. Bastyr EJ 3rd, Price KL, Bril V; MBBQ Study Group. Development and validity testing of the neuropathy total symptom score-6: questionnaire for the study of sensory symptoms of diabetic peripheral neuropathy. Clin Ther 2005;27:1278-94.

20. Fregni F, Gimenes R, Valle AC, Ferreira MJ, Rocha RR, Natalle L, et al. A randomized, sham-controlled, proof of principle study of transcranial direct current stimulation for the treatment of pain in fibromyalgia. Arthritis Rheum 2006;54:3988-98.

21. Roizenblatt S, Fregni F, Gimenez R, Wetzel T, Rigonatti SP, Tufik S, et al. Site-specific effects of transcranial direct current stimulation on sleep and pain in fibromyalgia: a randomized, sham-controlled study. Pain Pract 2007;7:297-306.

22. Busner J, Targum SD. The clinical global impressions scale: applying a research tool in clinical practice. Psychiatry (Edgmont) 2007;4:28-37.

23. Beck AT, Steer RA, Ball R, Ranieri W. Comparison of Beck Depression Inventories -IA and -II in psychiatric outpatients. J Pers Assess 1996;67:588-97.

24. Rossi S, Cappa SF, Babiloni C, Pasqualetti P, Miniussi C, Carducci F, et al. Prefrontal [correction of Prefontal] cortex in long-term memory: an "interference" approach using magnetic stimulation. Nat Neurosci 2001;4:948-52.

25. Herwig U, Satrapi P, Schonfeldt-Lecuona C. Using the international 10-20 EEG system for positioning of transcranial magnetic stimulation. Brain Topogr 2003;16:95-9.

26. Reidler JS, Mendonca ME, Santana MB, Wang X, Lenkinski R, Motta AF, et al. Effects of motor cortex modulation and descending inhibitory systems on pain thresholds in healthy subjects. J Pain 2012;13:450-8.

27. Poreisz C, Boros K, Antal A, Paulus W. Safety aspects of transcranial direct current stimulation concerning healthy subjects and patients. Brain Res Bull 2007;72:208-14.

28. Rasche D, Ruppolt M, Stippich C, Unterberg A, Tronnier VM. Motor cortex stimulation for long-term relief of chronic neuropathic pain: a 10 year experience. Pain 2006;121:43-52.

29. Shin HC, Chapin JK. Mapping the effects of motor cortex stimulation on somatosensory relay neurons in the rat thalamus: direct responses and afferent modulation. Brain Res Bull 1990;24:257-65.

30. Yoo WK, You SH, Ko MH, Kim ST, Park CH, Park JW, et al. High frequency rTMS modulation of the sensorimotor networks: behavioral changes and fMRI correlates. Neuroimage 2008;39:1886-95.

31. Nitsche MA, Paulus W. Sustained excitability elevations induced by transcranial DC motor cortex stimulation in humans. Neurology 2001;57:1899-901.

32. Lang N, Siebner HR, Ward NS, Lee L, Nitsche MA, Paulus W, et al. How does transcranial DC stimulation of the primary motor cortex alter regional neuronal activity in the human brain? Eur J Neurosci 2005;22:495-504.

33. Lefaucheur JP, Drouot X, Keravel Y, Nguyen JP. Pain relief induced by repetitive transcranial magnetic stimulation of precentral cortex. Neuroreport 2001;12:2963-5.

34. Antal A, Kriener N, Lang N, Boros K, Paulus W. Cathodal transcranial direct current stimulation of the visual cortex in the prophylactic treatment of migraine. Cephalalgia 2011;31:820-8.

35. Grundmann L, Rolke R, Nitsche MA, Pavlakovic G, Happe S, Treede RD, et al. Effects of transcranial direct current stimulation of the primary sensory cortex on somatosensory perception. Brain Stimul 2011;4:25360.

36. Arendt-Nielsen L, Bjerring P. Sensory and pain threshold characteristics to laser stimuli. J Neurol Neurosurg Psychiatry 1988;51:35-42. 